\title{
Solvothermal-induced phase transition and visible photocatalytic activity of nitrogen-doped titania
}

\author{
Jianjun Liu ${ }^{\mathrm{a}, *}$, Wei Qin ${ }^{\mathrm{a}}$, Shengli Zuo ${ }^{\mathrm{a}}$, Yingchun Yu${ }^{\mathrm{a}}$, Zhengping Hao ${ }^{\mathrm{b}}$ \\ a Faculty of Sciences, Beijing University of Chemical Technology, Beijing 100029, China \\ ${ }^{\mathrm{b}}$ Research Center for Eco-Environmental Sciences, Chinese Academy of Sciences, Beijing 100085, China
}

\section{A R T I C L E I N F O}

\section{Article history:}

Received 14 March 2008

Received in revised form 20 June 2008

Accepted 24 June 2008

Available online 1 July 2008

\section{Keywords:}

Nitrogen-doped titania

Solvothermal synthesis

Phase transition

Photocatalysis

Methyl orange

\begin{abstract}
A B S T R A C T
Nitrogen-doped titania nanoparticles consisting of pure anatase, pure rutile and bicrystallites (anatase + rutile and anatase + brookite) have been prepared in $\mathrm{TiCl}_{3}-\mathrm{HMT}$ (hexamethylene tetramine)alcohol solution under solvothermal process. The effect of the solvent type and amount of HMT as $\mathrm{pH}$ adjuster on the phase composition of titania and its visible photocatalytic activity for degradation to MO (methyl orange) was investigated. It is found that anatase gradually transferred to rutile with increase of carbon chain using methanol, ethanol, 1-propanol and 1-butanol as solvent. The pure anatase formed at the $\mathrm{pH}$ value of $1-2$, while bicrystalline titania (anatase + rutile and anatase + brookite) at that of 7-10 in the presence of methanol. The bicrystalline (anatase + brookite) titania have the best visible photocatalytic activity among all the samples. The -(NO) and -(NH) dopants with an $\mathrm{N}(1 \mathrm{~s})$ binding energy of $400 \mathrm{eV}$ may have positive effects on the visible light photocatalytic activity.
\end{abstract}

(c) 2008 Elsevier B.V. All rights reserved.

\section{Introduction}

Titania has a broad prospect for practical application in chemical and environmental industry as a heterogeneous photocatalyst and draws great worldwide researchers' attention due to its cheapness, nontoxicity, high photostability and powerful photocatalytic activity [1,2]. However, it is active only in the ultraviolet (UV) region because of its wide band gap (3.0-3.2 eV), which accounts for less than $5 \%$ of solar energy [3]. Recent studies have revealed that the shortcoming of $\mathrm{TiO}_{2}$ can be overcome by doping the nonmetal elements such as nitrogen [4-6], boron [7], sulfur [8], carbon [9], etc. For example, Asahi et al. [4] reported that $\mathrm{N}$-doped $\mathrm{TiO}_{2}$ showed photocatalytic activity for the decomposition of acetone and methylene blue in wavelengths up to $550 \mathrm{~nm}$ because the $\mathrm{N} 2 \mathrm{p}$ states contribute to the band gap narrowing by mixing with $\mathrm{O} 2 \mathrm{p}$ states by substitutional doping of nitrogen.

It is well known that three phases of titania including rutile, anatase and brookite, are found in nature, usually, only anatase and rutile are utilized as a photocatalyst. Both brookite and anatase are metastable phases, which can be converted to rutile phase after annealing [10]. As far as we know, there are many studies on anatase and rutile, but not much on brookite, since it is believed

\footnotetext{
* Corresponding author. Tel.: +86 10 64431056; fax: +86 1064434898 .

E-mail address: jjliu717@yahoo.com.cn (J. Liu).
}

that metastable brookite phase is difficult to synthesize. Now, pure brookite and polymorphs containing parts of brookite can be obtained by a hydrothermal or solvothermal method [11-13], however, only few reports about their photocatalytic properties have been published. There are many factors including the phase composition, crystallite size, morphology, specific surface area and energy band gap and so on that can influence the photocatalytic activity of $\mathrm{TiO}_{2}$. For example, Degussa P25 (made in Germany), a type of commercial nanosized titania which possesses a high photocatalytic activity, exists in polymorphs composed of about $80 \%$ anatase and $20 \%$ rutile. So it is highly important to study the relationship between phase composition and photocatalytic activity of $\mathrm{TiO}_{2}$ under different synthesis conditions.

Inorganic additives, such as alumina [14] and zirconia [15], have been successfully used to control the anatase/rutile phase transformation in the preparation of titania. However, it is difficult to remove the inorganic additives from the final products and/or avoid the possible influence of additives on photocatalytic activity. Recently, some controllable synthesis processes of crystalline nanosized titania have been developed without any inorganic additives. Luo et al. [16] synthesized some bicrystalline (anatase and rutile) and tricrystalline (anatase, rutile, and brookite) mesostructured titania by varying the solvent and cosolvent. Wang et al. [17] synthesized pure anatase, pure rutile and their mixed phases under alcohol solvothermal conditions. Yin et al. [18] reported the controllable synthesis of a variety of titania phase by hydrothermal treatment with titanium trichloride $\left(\mathrm{TiCl}_{3}\right)$ and various nitrogen 
sources. Qu et al. [19] synthesized nanosized titanium dioxide by a detonation route and discussed the stability of anatase and rutile from the differences in the surface energy of anatase and rutile at small crystallite sizes. In our work, $\mathrm{TiCl}_{3}$ and hexamethylene tetramine (HMT, $\mathrm{C}_{6} \mathrm{H}_{12} \mathrm{~N}_{4}$ ) were chosen as Ti and $\mathrm{N}$ sources in the preparation process of doped $\mathrm{TiO}_{2}$ with nitrogen, and the effects of reaction conditions such as the amount of HMT, type of solvents and $\mathrm{pH}$ value on the phase composition and photocatalytic activity were systematically investigated.

\section{Experimental}

\subsection{Solvothermal synthesis}

$\mathrm{TiCl}_{3}$ and HMT were used as starting materials. An amount of HMT, ranging from $2 \mathrm{~g}$ to $18 \mathrm{~g}$, was put into $50 \mathrm{~mL}$ aqueous solution containing $20 \mathrm{wt} \% \mathrm{TiCl}_{3}$, then were mixed with $50 \mathrm{~mL}$ of pure alcohol including methanol, ethanol, 1-propanol or 1-butanol. The final mixture was placed in a $200-\mathrm{mL}$ stainless-steel autoclave. Before the reaction, $\mathrm{pH}$ value of the reaction system (as initial $\mathrm{pH}$ in Table 1 ) was measured. The autoclave was heated and kept at $90^{\circ} \mathrm{C}$ for $1 \mathrm{~h}$ and then heated at $190^{\circ} \mathrm{C}$ for $2 \mathrm{~h}$. After the reaction, the sample was cooled naturally to room temperature, its $\mathrm{pH}$ value (as final $\mathrm{pH}$ in Table 1) was also measured. The products were separated by centrifugation, washed for several times with distilled water and ethanol to make sure no $\mathrm{Cl}^{-}$was detected (checked by $1 \% \mathrm{AgNO}_{3}$ solution), then vacuum dried at $60^{\circ} \mathrm{C}$ overnight. All the samples are yellow or yellowish powders in color. The $\mathrm{pH}$ values were measured before/after the reaction on a PHS-3C acidometer (made in China).

\subsection{Characterization of photocatalyst}

The phase composition of the products was determined on an X-ray diffractometer (XRD) (Rigaku D/MAX-2500) using Cu $\mathrm{K} \alpha$ radiation at $40 \mathrm{kV}$ work voltage and $200 \mathrm{~mA}$ work current. Microstructures were observed by a transmission electron microscope (TEM) (Hitachi H-800) at an accelerating voltage of $200 \mathrm{kV}$. The Brunauer-Emmett-Teller (BET) surface areas were obtained by measuring $\mathrm{N}_{2}$ adsorption isotherms at $77 \mathrm{~K}$ by using an ASAP2020 analyzer (Micromeritics). The absorption edges and band gaps of the products were determined using a UV-vis diffuse reflectance spectrophotometer (Shimadzu UV-vis 250 IPC). Nitrogen doping was confirmed by X-ray photoelectron spectrometry (XPS) (Thermo ESCA LAB 250).

\subsection{Evaluation of photocatalytic activity}

A 500-W Xe lamp was used as the light source and the visible wavelength was controlled through a 420-nm cut filter (LF420, China), which was hanged in a dark box and kept at about $15 \mathrm{~cm}$ above the liquid level. Aqueous suspensions of methyl orange (MO) dye $\left(100 \mathrm{~mL}\right.$, with an initial concentration of $\left.1.0 \times 10^{-4} \mathrm{M}\right)$ and photocatalyst powder $(50 \mathrm{mg})$ were placed in a beaker $(250 \mathrm{~mL})$. Prior to irradiation, the suspensions were magnetically stirred for 10 min under dark condition to establish an adsorption/desorption equilibrium between dye and photocatalyst surface. Under stirring, aliquots of a small amount of suspension (about $4 \mathrm{~mL}$ ) was taken out at every $5 \mathrm{~min}$ under irradiation conditions, then centrifuged and analyzed using a 721 spectrophotometer (made in China).

\subsection{Calculation of phase content}

The phase contents of the samples (Table 1 ) were calculated from the integrated intensities of anatase (101), rutile (1 110$)$, and brookite (121) peaks with the following formulas:

$$
\begin{gathered}
W_{\mathrm{A}}=\frac{k_{\mathrm{A}} A_{\mathrm{A}}}{k_{\mathrm{A}} A_{\mathrm{A}}+A_{\mathrm{R}}+k_{\mathrm{B}} A_{\mathrm{B}}} \\
W_{\mathrm{R}}=\frac{A_{\mathrm{R}}}{k_{\mathrm{A}} A_{\mathrm{A}}+A_{\mathrm{R}}+k_{\mathrm{B}} A_{\mathrm{B}}} \\
W_{\mathrm{B}}=\frac{k_{\mathrm{B}} A_{\mathrm{B}}}{k_{\mathrm{A}} A_{\mathrm{A}}+A_{\mathrm{R}}+k_{\mathrm{B}} A_{\mathrm{B}}}
\end{gathered}
$$

where $W_{A}, W_{R}$, and $W_{B}$ represent the weight fractions of anatase, rutile, and brookite, respectively. $A_{\mathrm{A}}, A_{\mathrm{R}}$, and $A_{\mathrm{B}}$ represent the integrated intensities of anatase (1 01$)$, rutile (1 10$)$, and brookite (1 21 ) peaks, respectively. According to the literature [16]: $k_{\mathrm{A}}=0.886$ and $k_{\mathrm{B}}=2.721$.

\section{Results and discussion}

\subsection{Effect of solvent type}

The XRD patterns of the catalyst samples prepared by solvothermal process in different alcohols with various carbon chain lengths $\left(\mathrm{C}_{m} \mathrm{H}_{2 m+1} \mathrm{OH}, m=1-4\right)$ are shown in Fig. 1 . As listed in Table 1, the phase contents of the samples can be obtained, according to the phase calculation formulas in Section 2.4. The contents of the anatase phase decrease in the order of sample nos. 1-4. Only the rutile phase can be observed, when the solvent is 1-butanol. That is to say, the content of the rutile phase increases with increasing length of carbon chain of the solvent until the pure rutile phase appeared in the 1-butanol media. We believe that this regular change is related to the molecular structure of solvent.

As shown in Table 1 , the initial $\mathrm{pH}$ values of reaction system decrease gradually in the order of samples 1 (methanol), 2 (ethanol), 3 (1-propanol), and 4 (1-butanol) that indicates the system acidity enhances. According to the reported mechanism [20], the chemical reactions responsible for the formation of $\mathrm{TiO}_{2}$ are as follows:

$$
\begin{aligned}
& \mathrm{TiCl}_{3}+x \mathrm{ROH} \rightarrow \mathrm{TiCl}_{3-x}(\mathrm{OR})_{x}\left(R=\mathrm{C}_{m} \mathrm{H}_{2 m+1}\right)+x \mathrm{HCl} \\
& \mathrm{TiCl}_{3-x}(\mathrm{OR})_{x}+\mathrm{O}_{2} \rightarrow \mathrm{Ti} \text { oxo species }+\mathrm{O}_{2}{ }^{-} \rightarrow \mathrm{TiO}_{2}
\end{aligned}
$$

In the process of alcoholysis, $\mathrm{TiCl}_{3-x}(\mathrm{OR})_{x}$ and hydrochloric acid form first, which makes the reaction system slightly acidic, then $\mathrm{TiCl}_{3-x}(\mathrm{OR})_{x}$ is assumed to be oxidized to form Ti(IV) oxo species, and finally $\mathrm{TiO}_{2}$ come into being. The reaction system containing $10 \mathrm{~g}$ HMT used as the precipitation reagent is alkaline and its initial

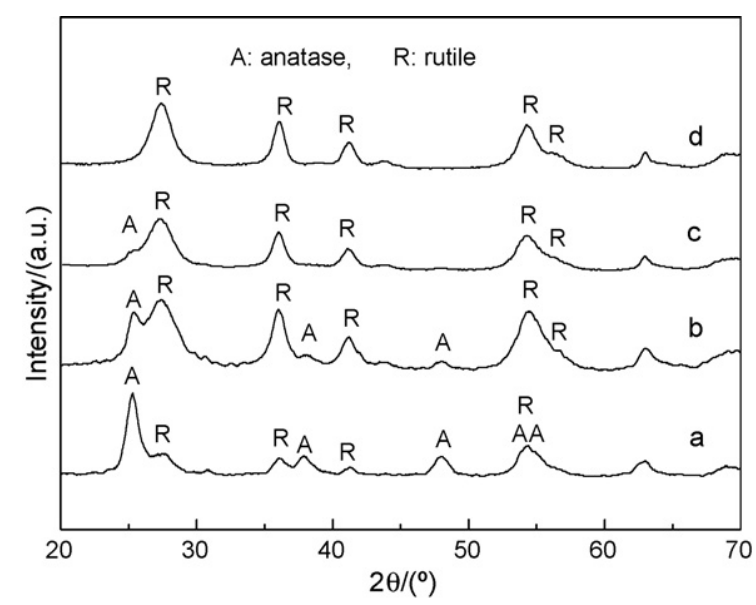

Fig. 1. XRD patterns of $\mathrm{TiO}_{2}$ synthesized under different solvothermal conditions (a) Methanol, no. 1; (b) ethanol, no. 2; (c) 1-propanol, no. 3; (d) 1-butanol, no. 4. 
Table 1

Effect of treatment solvent and HMT amount on physico-chemical properties of $\mathrm{TiO}_{2}$ samples

\begin{tabular}{|c|c|c|c|c|c|c|c|c|c|c|}
\hline \multirow[t]{2}{*}{ Sample no. ${ }^{\mathrm{a}}$} & \multirow[t]{2}{*}{ HMT amount (g) } & \multirow[t]{2}{*}{ Initial $\mathrm{pH}$} & \multirow[t]{2}{*}{ Final pH } & \multirow[t]{2}{*}{ Band gap (eV) } & \multirow[t]{2}{*}{$C / C_{0}{ }^{\mathrm{b}}$} & \multirow[t]{2}{*}{$\mathrm{N}$ doping amount (at.\%) } & \multirow[t]{2}{*}{ S.S.A $\left(\mathrm{m}^{2} \mathrm{~g}^{-1}\right)$} & \multicolumn{3}{|c|}{ Phase composition of $\mathrm{TiO}_{2}$} \\
\hline & & & & & & & & Anatase (\%) & Rutile (\%) & Brookite (\%) \\
\hline 1 & 10 & 9.15 & 10.05 & 2.92 & 0.35 & 1.83 & 228.9 & 80.5 & 19.5 & 0 \\
\hline 2 & 10 & 9.11 & 10.03 & 2.89 & 0.48 & 1.95 & 131.1 & 46.6 & 53.4 & 0 \\
\hline 3 & 10 & 9.08 & 10.07 & 3.00 & 0.70 & 2.13 & 142.5 & 22.8 & 77.2 & 0 \\
\hline 4 & 10 & 9.01 & 9.97 & 2.91 & 0.77 & 2.06 & 154.4 & 0 & 100 & 0 \\
\hline 5 & 2 & 1.03 & 2.88 & 3.01 & 0.53 & 2.88 & 166.7 & 100 & 0 & 0 \\
\hline 6 & 6 & 7.24 & 9.52 & 2.94 & 0.57 & 2.03 & 160.8 & 47.0 & 53.0 & 0 \\
\hline 7 & 14 & 10.21 & 10.31 & 3.16 & 0.28 & 2.39 & 197.6 & 50.4 & 0 & 49.6 \\
\hline 8 & 18 & 10.27 & 10.30 & 2.99 & 0.13 & 2.41 & 124.4 & 26.9 & 0 & 73.1 \\
\hline
\end{tabular}

a In sample nos. 1, 5, 6, 7 and 8 methanol is used as solvent, while in sample nos. 2, 3 and 4 ethanol, 1-propanol and 1-butanol are used as solvents, respectively.

b The ratio between $C$ of remaining $\mathrm{MO}$ concentration at $50 \mathrm{~min}$ and initial $C_{0}$.

$\mathrm{pH}$ value is about 9. It is noticeable that it decreases a little with increasing carbon chain length of the solvent.

We believe that, as the number of carbon chain in alcohol increases, the $\mathrm{Cl}$ atom in $\mathrm{TiCl}_{3}$ is substituted by an alkoxy group $(-\mathrm{OR})$ which contains longer carbon chain and forms a $\mathrm{TiCl}_{3-x}(\mathrm{OR})_{x}$ complex which has a higher steric hindrance. Thus, more chlorine stays in the system to form more $\mathrm{HCl}$, which dissolves easily in water, so the acidity of the system increases with the number of carbon chain, as listed in Table 1 . The Ti(III) complex has the formula $\mathrm{TiCl}_{3-x}(\mathrm{OR})_{x}$, where $x(=1-3)$ is concerned with the acidity and concentration of $\mathrm{Cl}^{-}$, i.e., the higher the acidity and $\left[\mathrm{Cl}^{-}\right]$, the bigger is $x$. When the acidity is lower, the number of OR ligand in $\mathrm{TiCl}_{3-x}(\mathrm{OR})_{x}$ is less, which could be beneficial for the formation of the anatase phase with edge-shared bonding. In contrast to this, the formation of rutile phase with corner-shared bonding could be at higher acidity and concentration of $\left[\mathrm{Cl}^{-}\right]$. This viewpoint was confirmed by Chen et al. by using $\mathrm{SnCl}_{4}, \mathrm{NaCl}$ and $\mathrm{NH}_{4} \mathrm{Cl}$ as mineralizers to prepare nanosize titania under hydrothermal conditions [21]. It can been seen from Table 1 that all final $\mathrm{pH}$ values increase after the reaction, because HMT $\left(\mathrm{C}_{6} \mathrm{H}_{12} \mathrm{~N}_{4}\right)$ was decomposed to produce $\mathrm{NH}_{3}$ according to the following reaction equations:

$\mathrm{C}_{6} \mathrm{H}_{12} \mathrm{~N}_{4}+6 \mathrm{H}_{2} \mathrm{O} \rightarrow 6 \mathrm{HCHO}+4 \mathrm{NH}_{3}$

$\mathrm{NH}_{3}+\mathrm{H}_{2} \mathrm{O} \rightleftharpoons \mathrm{NH}_{4}^{+}+\mathrm{OH}^{-}$

The synthetic process is actually in a mixed water-alcohol medium, not in a pure alcohol-based medium, since Ti source is a $20 \% \mathrm{TiCl}_{3}$ aqueous solution. So $\mathrm{NH}_{3}$ produced by HMT dissolves in water and alcohol, which makes $\mathrm{pH}$ values increase. But the change in final $\mathrm{pH}$ values in Table 1 is irregular. This is attributed to the dependence of final pH on the different $\mathrm{NH}_{3}$ solubility and the amount of $\mathrm{NH}_{3}$ released by decomposing the HMT in the various water-alcohol media.

The photocatalytic degradation curves and remaining concentrations of $\mathrm{MO}$ on $\mathrm{TiO}_{2}$ synthesized under different solvothermal conditions are shown in Fig. 2 and Table $1\left(C / C_{0}\right)$, respectively. The $\mathrm{MO}$ dye can hardly be degraded by undoped $\mathrm{TiO}_{2}$ under visible irradiation. It is obvious that the doped $\mathrm{TiO}_{2}$ samples synthesized in methanol and ethanol show higher photocatalytic activities. Both of them are bicrystalline (rutile and anatase). The sample no. 1 synthesized in methanol (no. 1), in particular, has highest photocatalytic activity for the photodegradation of MO. As listed in Table 1, the sample no. 1 shows the largest specific surface area of $228.9 \mathrm{~m}^{2} / \mathrm{g}$ and the lowest N $1 \mathrm{~s}$ doping amount of 1.83 at.\%, and contains $19.5 \%$ of rutile and $80.5 \%$ of anatase. It is widely accepted that the mixed phase of titania is beneficial for reducing the recombinations of photogenerated electrons and holes, and this synergistic effect always results in an enhancement of photocatalytic activity [22,23]. Degussa P25, consisting of about $80 \%$ of anatase and $20 \%$ of rutile, is extensively studied as a standard titania photo- catalyst partially because of its high photocatalytic activity which is profited from the mixed phase of anatase and rutile. So in our further research, all the $\mathrm{TiO}_{2}$ samples were synthesized under the solvothermal condition of methanol, and $\mathrm{TiO}_{2}$ samples with higher visible photocatalytic activities are expected to be obtained by varying other reaction conditions.

\subsection{Effect of HMT amount}

The XRD patterns of $\mathrm{TiO}_{2}$ samples prepared by adding different amounts of HMT in the methanol media are shown in Fig. 3. With increasing amount of alkaline HMT, the initial $\mathrm{pH}$ values gradually increase from 1.03 to 7.24 , with the variety of the reaction environment from acidic to alkaline, and pure anatase- $\mathrm{TiO}_{2}$ sample transfers to a rutile-anatase mixture, as shown in Table 1 . It seems reasonable to believe that $\mathrm{TiO}_{2}$ samples prepared under acidic condition are preferred to form anatase, while under alkaline condition, to form rutile, although the detail is not clear yet. Similar results were observed in the $\mathrm{TiCl}_{3}$-HMT-aqueous solution via solvothermal process reported by Aita et al. [24]. When the initial $\mathrm{pH}$ value increases to 10 , rutile vanishes and brookite appears and increases with increasing amount of HMT.

When the reactions are at ambient condition, $\mathrm{NH}_{3}$ released by decomposing the HMT reaches the equilibrium solubility in water, therefore no corresponding increase of $\mathrm{pH}$ value occurs, even though the release of $\mathrm{NH}_{3}$ also increases with the increase of HMT. In the process of heating procedures in the confined autoclave, the increased pressure of the system will lead to the increase of solubility of $\mathrm{NH}_{3}$ in alcohol-water and $\mathrm{pH}$ value. It can be seen in

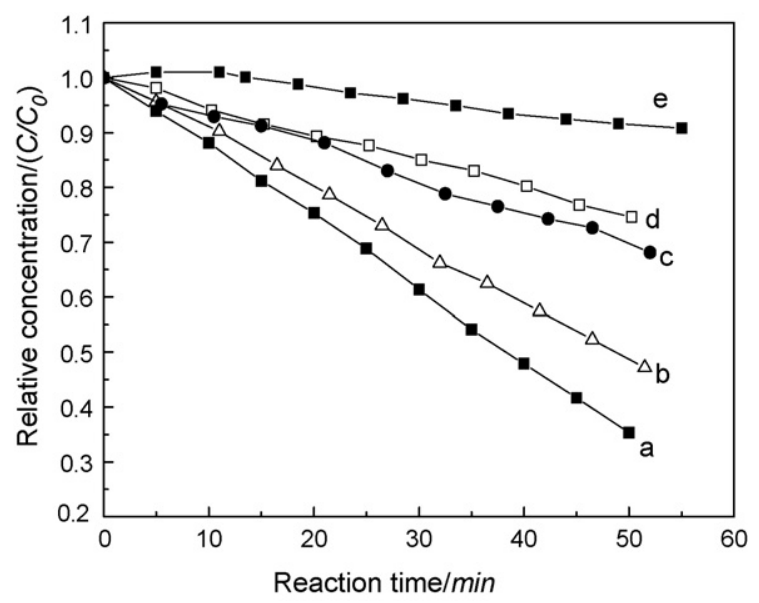

Fig. 2. Curves of photocatalytic activity of $\mathrm{TiO}_{2}$ synthesized under different solvothermal conditions: (a) methanol, no. 1; (b) ethanol, no. 2; (c) 1-propanol, no. 3; (d) 1-butanol, no. 4; (e) P25. 


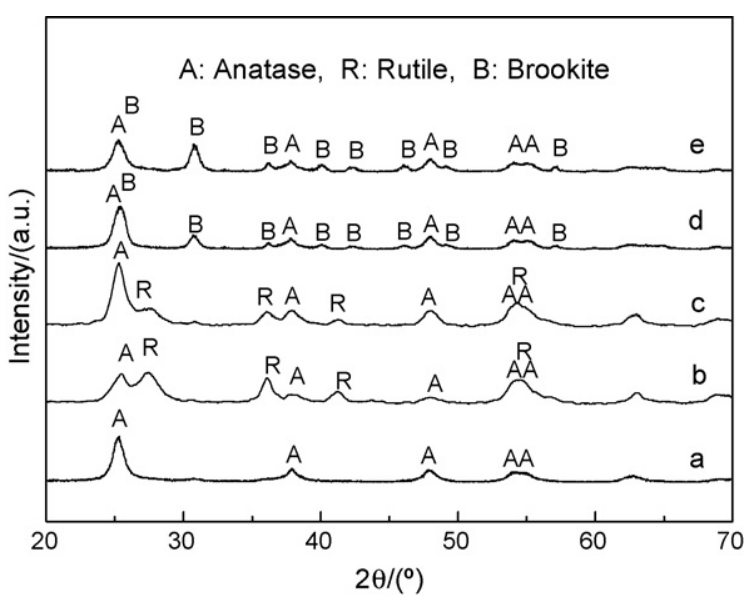

Fig. 3. XRD patterns of $\mathrm{TiO}_{2}$ synthesized by adding different amounts of HMT: (a) 2 g, no. 5; (b) 6 g, no. 6; (c) 10 g, no. 1; (d) 14 g, no. 7; (e) 18 g, no. 8.

Table 1 that the alkali conditions are beneficial for the formation of brookite. This view is in agreement with the results of Yao et al. [25], who reported the synthesis of brookite under the hydrothermal conditions $\left(\mathrm{pH}_{\text {initial }} 10-12\right)$ using $\mathrm{TiCl}_{4}$ and $\mathrm{NaOH}$ as precursors.

The TEM images of the samples prepared in $\mathrm{TiCl}_{3}-\mathrm{HMT}$ methanol solutions with different amounts of HMT are shown in Fig. 4. It is obvious that the morphology of $\mathrm{TiO}_{2}$ particles is different. The bicrystalline $\mathrm{TiO}_{2}$ (anatse + rutile) obtained at $\mathrm{pH} 7$ consists of large spherical particles of $10-20 \mathrm{~nm}$ and acicular particles of $40-50 \mathrm{~nm}$ in length, $6-8 \mathrm{~nm}$ in width (Fig. 4(a)), while the bicrystalline $\mathrm{TiO}_{2}$ (anatse+brookite) prepared at $\mathrm{pH} 10$ shows smaller spherical particles of 5-15 nm and flake particles of $10-30 \mathrm{~nm}$ (Fig. 4(b)). The different morphology of particles can be correlated with crystal growths of the three $\mathrm{TiO}_{2}$ phases at different conditions of $\mathrm{pH}$ value.

The photocatalytic degradation curves and remaining concentrations of $\mathrm{MO}$ on the doped $\mathrm{TiO}_{2}$ prepared by adding different amounts of HMT are shown in Fig. 5 and Table $1\left(C / C_{0}\right)$, respectively. It can be seen that the all samples exhibit good photocatalytic degradation activities compared with the undoped $\mathrm{P} 25 \mathrm{TiO}_{2}$. Furthermore, the bicrystalline (anatase + brookite) titania (nos. 7 and 8 ) have more higher photocatalytic activities than that of pure anatase (no.5) and bicrystalline (anatase + rutile) ones (nos. 1 and 6) in which the sample (no. 8) consisting of more brookite (73.1\%) and less anatase (26.9\%) has the best photocatalytic activity, although its specific surface area of $124.4 \mathrm{~m}^{2} / \mathrm{g}$ is the smallest in all the samples (Table 1). These results suggest that the existence of more brookite phase can improve the photocatalytic activity under the condition of the nearly same $\mathrm{N}$-doping status $(\sim 400 \mathrm{eV})$ and doping amount

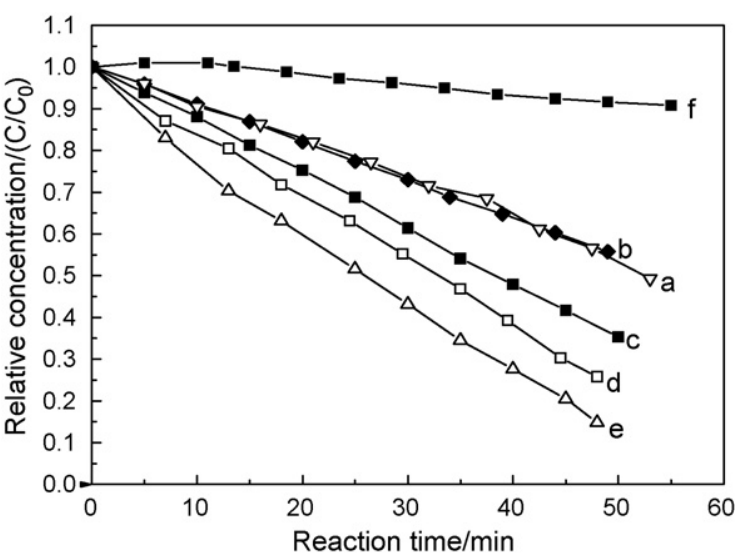

Fig. 5. Curves of photocatalytic activity of $\mathrm{TiO}_{2}$ sample synthesized by adding different amounts of HMT: (a) 2 g, no. 5; (b) 6 g, no. 6; (c) 10 g, no. 1; (d) 14 g, no. 7; (e) $18 \mathrm{~g}$, no. 8; (f) P25.

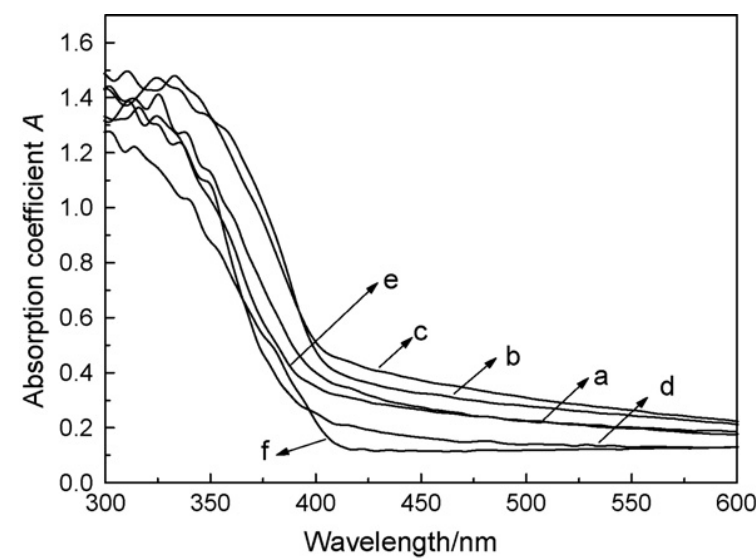

Fig. 6. UV-vis spectra of $\mathrm{TiO}_{2}$ synthesized by adding different amounts of HMT: (a) 2 g, no. 5; (b) 6 g, no. 6, (c) 10 g, no. 1; (d) 14 g, no. 7; (e) 18 g, no. 8; (f) P25.

( $\sim 2.4$ at.\%) for samples of nos. 7 and 8 . Although the details of this have not yet been clarified, it is generally accepted that the synergistic effect of mixed phases of $\mathrm{TiO}_{2}$ can enhance the photocatalytic activity [26,27].

The UV-vis spectra of $\mathrm{P} 25$ and the $\mathrm{TiO}_{2}$ powders prepared with different amount of HMT are shown in Fig. 6. The N-doped $\mathrm{TiO}_{2}$ samples, because of their yellow colors, display drastic and stronger photoabsorption in the range of wavelengths from 400 to $550 \mathrm{~nm}$ than that of the white color P-25. According to the absorption edge of nitrogen-doped $\mathrm{TiO}_{2}$ shown in Fig. 6, the band gaps of different
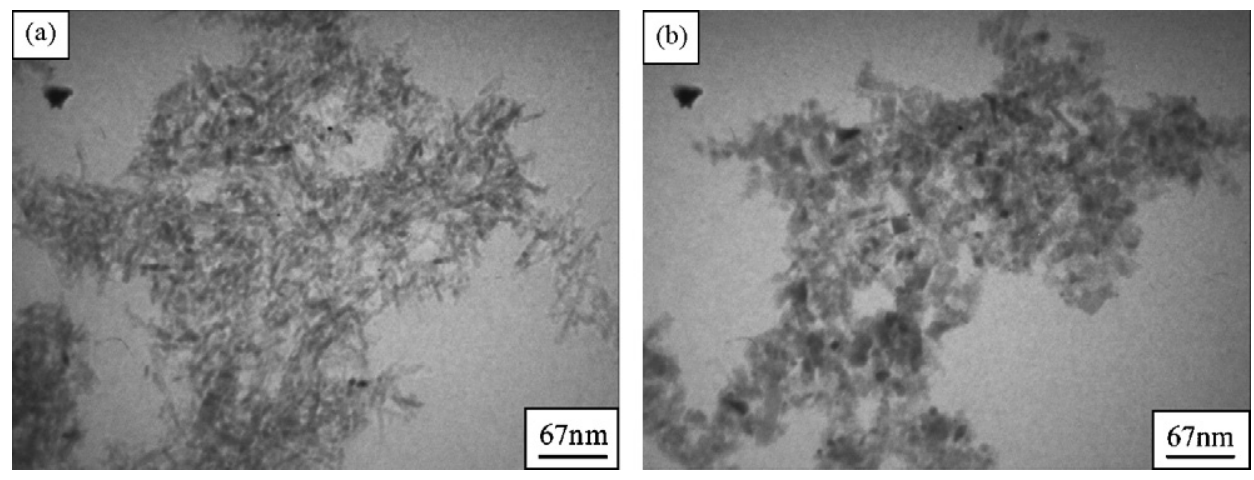

Fig. 4. TEM images of $\mathrm{TiO}_{2}$ synthesized by adding different amounts of HMT: (a) $6 \mathrm{~g}$, no. 6, (b) $14 \mathrm{~g}$, no. 7 . 
samples are estimated from the plot of $\alpha^{1 / 2}$ versus photon energy $(h \nu)$ and are listed in Table 1. For pure anatase, rutile and brookite, the intrinsic band gap is $3.2 \mathrm{eV}, 3.0 \mathrm{eV}$ and $3.4 \mathrm{eV}[28,29]$, respectively. Because of the well-known quantum size effect the band gaps are a strong function of titania particle size less than $10 \mathrm{~nm}$. Thus, as shown in Fig. 4, smaller the particle size, higher is the band gap. However, the varying degrees of decrease in band gaps can be seen in all the nitrogen-doped $\mathrm{TiO}_{2}$ samples compared with those in pure (nos. 4 and 5) and mixed phases (nos. 1-3 and 6-8) of titania, as shown in Table 1 . The results indicate that the nitrogen doping has very important effect on the decrease of the band gap of $\mathrm{TiO}_{2}$ and exhibits higher visible photocatalytic activity than that of undoped $\mathrm{P}-25 \mathrm{TiO}_{2}$.

\subsection{Effect of $N$-doping}

XPS analysis shows that the binding energy of $\mathrm{N} 1 \mathrm{~s}$ in our doped samples synthesized in methanol is only at about $400 \mathrm{eV}$ in Fig. 7. Asahi et al. [4] analyzed their $\mathrm{N}$-doped $\mathrm{TiO}_{2}$ with XPS and observed three $\mathrm{N} 1 \mathrm{~s}$ peaks with the binding energies of $402 \mathrm{eV}, 400 \mathrm{eV}$ and $396 \mathrm{eV}$. They assigned these peaks as the formation of Ti-N bonding $(396 \mathrm{eV})$ and molecularly chemisorbed $\mathrm{N}_{2}$ ( $400 \mathrm{eV}$ and $\left.402 \mathrm{eV}\right)$, in which the only $\mathrm{N}$-doping at $396 \mathrm{eV}$ is responsible for visible light sensitization. It is widely known that molecular $\mathrm{N}_{2}$ is not chemisorbed on metal oxides such as $\mathrm{TiO}_{2}$ at room temperature. So the assignment of the $\mathrm{N} 1 \mathrm{~s}$ peak at $400 \mathrm{eV}$ and $402 \mathrm{eV}$ to molecular $\mathrm{N}_{2}$ is implausible. Up to now, as the visible light activity of $\mathrm{N}$-doped $\mathrm{TiO}_{2}$ involving only $396 \mathrm{eV} \mathrm{N} 1$ s state has not been demonstrated, the contribution of $\mathrm{Ti}-\mathrm{N}$ bonding to the visible light sensitization of $\mathrm{TiO}_{2}$ remains in doubt. The results of Diwald et al. [30] rather indicate negative contribution of $\mathrm{Ti}-\mathrm{N}$ bonding to photocatalytic activity. According to the reports [31,32], $\mathrm{N} 1 \mathrm{~s}$ state at $400 \mathrm{eV}$ in our doped samples of $\mathrm{TiO}_{2}$ samples by the solvothermal method is in an oxidized state similar to -(NO) and interstitially dopant $-(\mathrm{NH})$

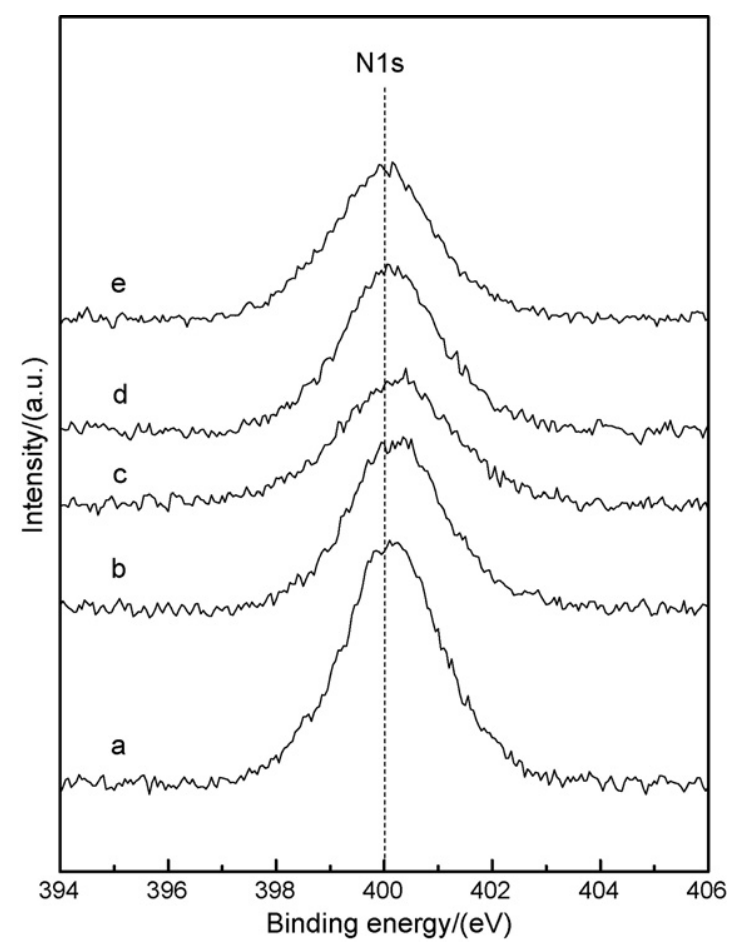

Fig. 7. XPS patterns of $\mathrm{N} 1 \mathrm{~s}$ spectra for the doped $\mathrm{TiO}_{2}$ synthesized by adding different amounts of HMT: (a) 2 g, no. 5; (b) 6 g, no. 6; (c) $10 \mathrm{~g}$, no. 1; (d) $14 \mathrm{~g}$, no. 7; (e) $18 \mathrm{~g}$, no. 8 . from HMT, which can be attributed to the doping status of $\mathrm{N} 1 \mathrm{~s}(\mathrm{II})$ at 398.8-401.2 eV classified by Chen et al. [33]. The -(NO) and -(NH) dopants may have positive effects on the visible light photocatalytic activity.

According to the integrated intensity of the peaks around $400 \mathrm{eV}$, the $\mathrm{N} 1 \mathrm{~s}$ doping amount in our doped samples of $\mathrm{TiO}_{2}$ samples could be calculated, as shown in Table 1 . It is irregular variety of the $\mathrm{N} 1 \mathrm{~s}$ doping amount for the doped $\mathrm{TiO}_{2}$ samples (nos. 1-8) independent of the adding amount of HMT, such as no. 5 has the highest N 1s doping amount although with the lowest adding amount of HMT of $2 \mathrm{~g}$ that indicate the other factor such as $\mathrm{pH}$ value, phase composition, etc. may also affect the $\mathrm{N} 1 \mathrm{~s}$ doping amount of $\mathrm{TiO}_{2}$ samples.

\section{Conclusions}

(1) The anatase gradually transferred to rutile with the increase of carbon chain of methanol, ethanol, 1-propanol and 1-butanol as the solvent under solvothermal conditions. The bicrystalline $\mathrm{TiO}_{2}$ consisting of $19.5 \%$ of rutile and $80.5 \%$ of anatase prepared in methanol has the highest photocatalytic activity for the photocatalytic degradation of MO.

(2) It is found that anatase is easier to form at acidic condition, but bicrystallites (anatase + rutile and anatase + brookite) at basic condition, using methanol as solvent. The bicrystalline $\mathrm{TiO}_{2}$ containing anatase and brookite possesses the highest photocatalytic activity among all the samples.

\section{References}

[1] K. Masaaki, M. Masaya, U. Michio, A. Masakazu, Recent developments in titanium oxide-based photocatalysts, Appl. Catal. A 325 (2007) 1-14.

[2] A. Fujishima, T.N. Rao, D.A. Tryk, Titanium dioxide photocatalysis, J. Photochem. Photobiol. C1 (2000) 1-21.

[3] S. Yin, Q.W. Zhang, F. Saito, T. Sato, Preparation of visible light-activated titania photocatalyst by mechanochemical method, Chem. Lett. 32 (2003) 358-359.

[4] R. Asahi, T. Morikawa, T. Ohwaki, K. Aoki, Y. Taga, Visible-light photocatalysis in nitrogen-doped titanium oxides, Science 293 (2001) 269-271.

[5] D. Klauson, E. Portjanskaja, S. Preis, Visible light-assisted photocatalytic oxidation of organic pollutants using nitrogen-doped titania, Environ. Chem. Lett. 6 (2008) 35-39.

[6] Q. Li, R. Xie, E.A. Mintz, J.K. Shang, Enhanced visible-light photocatalytic degradation of humic acid by palladium-modified nitrogen-doped titanium dioxide, J. Am. Chem. Soc. 90 (2007) 3863-3868.

[7] A.Zaleska, J.W. Sobczak, E. Grabowska, J. Hupka, Preparation and photocatalytic activity of boron-modified $\mathrm{TiO}_{2}$ under UV and visible light, Appl. Catal. B 78 (2008) 92-100.

[8] J.H. Xu, J. Li, W.L. Dai, Y. Cao, H. Li, K. Fan, Simple fabrication of twist-like helix N,S-codoped titania photocatalyst with visible-light response, Appl. Catal. B 79 (2008) 72-80.

[9] M.S. Wong, S.W. Hsu, K.K. Rao, C.P. Kumar, Influence of crystallinity and carbon content on visible light photocatalysis of carbon doped titania thin films, J. Mol. Catal. A 279 (2008) 20-26.

[10] X. Bokhimi, A. Morales, M. Aguilar, J.A. Toledo-Antonio, F. Pedraza, Local order in titania polymorphs, Int. J. Hydrogen Energy 26 (2001) 1279-1287.

[11] K. Tomita, V. Petrykin, M. Kobayashi, M. Shiro, M. Yoshimura, M. Kakihana, A water-soluble titanium complex for the selective synthesis of nanocrystalline brookite, rutile, and anatase by a hydrothermal method, Angew. Chem. Int. Ed. 45 (2006) 2378-2381.

[12] S. Yin, Y. Aita, M. Komatsu, T. Sato, Visible-light-induced photocatalytic activity of $\mathrm{TiO}_{2-x} \mathrm{~N}_{y}$ prepared by solvothermal process in urea-alcohol system, J. Eur. Ceram. Soc. 26 (2006) 2735-2742.

[13] J.P. Wei, J.F. Yao, X.Y. Zhang, W. Zhu, H.T. Wang, M.J. Rhodes, Hydrothermal growth of titania nanostructures with tunable phase and shape, Mater. Lett. 61 (2007) 4610-4613.

[14] J. Yang, Y.X. Huang, J.M. Ferreira, Inhibitory effect of alumina additive on the titania phase transformation of a sol-gel-derived powder, J. Mater. Sci. Lett. 16 (1997) 1933-1935.

[15] J. Yang, J.M. Ferreira, On the titania phase transition by zirconia additive in a sol-gel-derived powder, Mater. Res. Bull. 33 (1998) 389-394.

[16] H. Luo, C. Wang, Y. Yan, Synthesis of mesostructured titania with controlled crystalline framework, Chem. Mater. 15 (2003) 3841-3846.

[17] C. Wang, Z. Deng, G. Zhang, S. Fan, Y. Li, Synthesis of nanocrystalline $\mathrm{TiO}_{2}$ in alcohols, Powder Technol. 125 (2002) 39-44.

[18] S. Yin, K. Ihara, Y. Aita, M. Komatsu, T. Sato, Visible-light induced photocatalytic activity of $\mathrm{TiO}_{2-x} \mathrm{~A}_{y}(\mathrm{~A}=\mathrm{N}, \mathrm{S})$ prepared by precipitation route, J. Photochem. Photobiol. A 179 (2006) 105-114. 
[19] Y.D. Qu, X.J. Li, X.H. Wang, D.H. Liu, Detonation synthesis of nanosized titanium dioxide powders, Nanotechnology 18 (2007), 205602/1-5.

[20] E. Hosono, S. Fujihara, K. Kakiuchi, H. Imai, Growth of submicrometer-scale rectangular parallelepiped rutile $\mathrm{TiO}_{2}$ films in aqueous $\mathrm{TiCl}_{3}$ solutions under hydrothermal conditions, J. Am. Chem. Soc. 126 (2004) 7790-7791.

[21] H.M. Cheng, J.M. Ma, Z.G. Zhao, L.M. Qi, Hydrothermal preparation of uniform nanosize rutile and anatase particles, Chem. Mater. 7 (1995) 663-671.

[22] J.C. Yu, J. Yu, W. Ho, L. Zhang, Preparation of highly photocatalytic active nano-sized $\mathrm{TiO}_{2}$ particles via ultrasonic irradiation, Chem. Commun. 1 (2001) 1942-1944.

[23] T. Ohno, K. Tokieda, S. Higashida, M. Matsumura, Synergism between rutile and anatase $\mathrm{TiO}_{2}$ particles in photocatalytic oxidation of naphthalene, Appl. Catal. A 244 (2003) 383-391.

[24] Y. Aita, M. Komatsu, S. Yin, T. Sato, Phase-compositional control and visible light photocatalytic activity of nitrogen-doped titania via solvothermal process, J. Solid State Chem. 177 (2004) 3235-3238.

[25] C. Yao, G. Yang, X.P. Lin, X.J. Yang, L.D. Lu, X. Wang, Effect of hydrothermal conditions on the microstructure of brookite titanium dioxide, Chin. J. Inorg. Chem. 21 (2005) 1821-1826 (in Chinese).

[26] H. Kominami, Y. Ishii, M. Kohno, S. Konishi, Y. Kera, B. Ohtani, Nanocrystalline brookite-type titanium(IV) oxide photocatalysts prepared by a solvothermal method: correlation between their physical properties and photocatalytic activities, Catal. Lett. 91 (2003) 41-47.

[27] J. Li, C. Tang, D. Li, H. Haneda, T. Ishigaki, Monodispersed spherical particles of brookite-type $\mathrm{TiO}_{2}$ : synthesis, characterization, and photocatalytic property, J. Am. Ceram. Soc. 87 (2004) 1358-1361.

[28] A.L. Linsebigler, G.Q. Lu, T. Yates Jr., Photocatalysis on $\mathrm{TiO}_{2}$ surfaces: principles, mechanisms, and selected results, Chem. Rev. 95 (1995) 735-758.

[29] M. Koelsch, S. Cassaignon, J.F. Guillemoles, J.P. Jolivet, Comparison of optical and electrochemical properties of anatase and brookite $\mathrm{TiO}_{2}$ synthesized by the sol-gel method, Thin Solid Films 403 (2002) 312-319.

[30] O. Diwald, T.L. Thompson, E.G. Goralski, S.D. Walck, J.T. Yates, The effect of nitrogen ion implantation on the photoactivity of $\mathrm{TiO}_{2}$ rutile single crystals, J. Phys. Chem. B 108 (2004) 52-57.

[31] S. Sato, R. Nakamura, S. Abe, Visible-light sensitization of $\mathrm{TiO}_{2}$ photocatalysts by wet-method N doping. Appl. Catal. A 284 (2005) 131-137.

[32] O. Diwald, T.L. Thompson, T. Zubkov, E.G. Goralski, S.D. Walck, J.T. Yates, Photochemical activity of nitrogen-doped rutile $\mathrm{TiO}_{2}\left(\begin{array}{ll}1 & 1\end{array}\right)$ in visible light, J. Phys. Chem. B 108 (2004) 6004-6008.

[33] C. Chen, H. Bai, C. Chang, Effect of plasma processing gas composition on the nitrogen-doping status and visible light photocatalysis of $\mathrm{TiO}_{2}$, J. Phys. Chem. C 111 (2007) 15228-15235. 